\title{
Core electron deformation in silicon from powder $X$-ray diffraction
}

Kasper Tolborg ${ }^{1}$, Mads R. V. Jørgensen ${ }^{1}$, Hidetaka Kasai ${ }^{2}$, Jacob Becker ${ }^{1}$, Ann-Christin Dippel ${ }^{3}$, Jens Als-Nielsen ${ }^{4}$, Bo B. Iversen ${ }^{1}$

${ }^{1}$ Center For Materials Crystallography, Department Of Chemistry, Aarhus University, Aarhus, Denmark, ${ }^{2}$ Faculty of Pure and Applied Sciences, CIRfSE and TIMS, University of Tsukuba, Tsukuba, Japan, ${ }^{3}$ PETRA III, Deutsches Elektronen-Synchrotron - DESY, Hamburg, Germany, ${ }^{4}$ Niels Bohr Institute, University of Copenhagen, Copenhagen, Denmark E-mail: tolborg@chem.au.dk

Traditionally Single Crystal X-Ray Diffraction (SCXRD) is used to obtain structure factor amplitudes for Electron Density (ED) modelling in crystalline materials. However, for SCXRD there are some pertinent issues with systematic errors such as extinction, absorption and scaling between detector frames. All three issues can be at least partially solved by performing Powder X-Ray Diffraction (PXRD). Two major issues are, however, present in PXRD; peak overlap and background treatment.

Recently, we have shown that PXRD can be a valuable alternative to SCXRD for ED determination in high symmetry inorganic materials, and the subtle deformations in core ED upon bond formation have been determined for diamond, silicon and cubic boron nitride. For silicon and diamond, the extracted structure factors were shown to be of comparable precision and accuracy to the dynamical Pendellösung data $[1,2]$. This was made possible by performing diffraction in vacuum with the first version of our custom built Aarhus Vacuum Imaging plate Diffractometer (AVID) with a sample-to-detector distance of 300 $\mathrm{mm}$. We have now finished commissioning of a new version of AVID with sample-to-detector distance increased to 1200 $\mathrm{mm}$, which is shown to give an improvement in signal-to-background ratio and instrumental peak broadening by a factor of three due to the differences in radial dependences of coherent and incoherent scattering [3].

Benchmark PXRD data on silicon has been collected on the new AVID showing Bragg peaks to an unprecedented resolution of $\sin \theta / \lambda \sim 2.4 \AA^{-1}$ at $100 \mathrm{~K}$. Data are modelled using a combined HC-Rietveld method, where overlapping reflections are partitioned based on the Hansen-Coppens multipole model, which reduces bias towards the independent atom model. The extracted structure factors are compared with the previous AVID and shown to be of significantly improved precision and accuracy at high angles. These are used for modelling the ED in silicon including deformations in the core ED with improved accuracy compared to previous studies due to the improved data resolution.

[1] Bindzus, N. et al. (2014). Acta Cryst. A70, 39-48

[2] Wahlberg, N. et al. (2016). Acta Cryst. A72, 28-35

[3] Tolborg, K. et al. (2017) Submitted to Acta Cryst. B

Keywords: Powder X-ray diffraction, core electron deformation, silicon 